\title{
Malignant Mesenchymal Tumor of the Prostate: A Case Report
}

\author{
Mustafa KANDAZ, ${ }^{1}$ Ozan Cem GÜLER, ${ }^{1}$ Elanur KARAMAN, ${ }^{2}$ Sevdegül MUNGAN, ${ }^{3}$ Adnan YÖNEY ${ }^{1}$
}

'Department of Radiation Oncology, KTU Medical Faculty, Trabzon-Turkey

${ }^{2}$ Department of Medical Oncology, KTU Medical Faculty, Trabzon-Turkey

${ }^{3}$ Department of Pathology, KTU Medical Faculty, Trabzon-Turkey

\begin{abstract}
SUMMARY
Nonepithelial tumors of the prostate represent $<1 \%$ of all prostate tumors. A rare soft tissue sarcoma is common in the lower extremities and can occur anywhere in the body. Malignant mesenchymal tumors of the prostate are rarely observed. The histogenesis of malignant mesenchymal tumors that have totipotential differentiation capacity and arise from primitive mesenchymal cells is still unknown. These are malignant soft tissue sarcomas including $\geq 2$ different mesenchymal elements in addition to the fibrosarcomatous elements. This type of tumors can occur anywhere within the body; the most frequently reported sites are the retroperitoneal cavity and lower extremity. They may occur in the heart, mediastinum, pleura, liver, eyes, bones, larynx, thyroid, testis, uterus, and bladder. The malignant potential is high and the progression of these tumors is rapid. There is mass in the prostate; however, when the prostate-specific antigen levels are normal, differential diagnoses should be considered. Although the main treatment for this disease is surgery, chemotherapy and/or radiotherapy can be applied. We present a rare case of malignant mesenchymal tumor of the prostate.

Keywords: Malignant mesenchymal tumor; prostate.

Copyright $\odot$ 2017, Turkish Society for Radiation Oncology
\end{abstract}

\section{Introduction}

The most common cancer in men is the epithelial prostate cancer. The rarely seen soft tissue tumors are a heterogeneous tumor family consisting of different subgroups, which have different biological and natural courses and responses to treatment. The histogenesis of malignant mesenchymal tumors that have totipotential differentiation capacity and arise from primitive mesenchymal cells is still unknown. These are malignant soft tissue sarcomas including $\geq 2$ different mesenchymal elements in addition to the fibrosarcomatous elements. This type of tumors can occur anywhere within the body; the most frequently reported sites are the retroperitoneal cavity and lower extremity. They may occur in the heart, mediastinum, pleura, liver, eyes, bones, larynx, thyroid, testis, uterus, and bladder.

Nonepithelial tumors of the prostate represent $<1 \%$ of all prostate cancers.[1] The prognosis of nonepithelial tumors of the prostate is worse compared with epithelial tumors of the prostate and their treatment methods are also different. Leiomyosarcoma is the most common malignant mesenchymal lesion of the prostate observed in adults.[2] The correct diagnosis need to be made to this case through multidisciplinary approach. As there are a limited number of cases of malignant mesenchymal tumors of the prostate in the literature, we presented this case. 

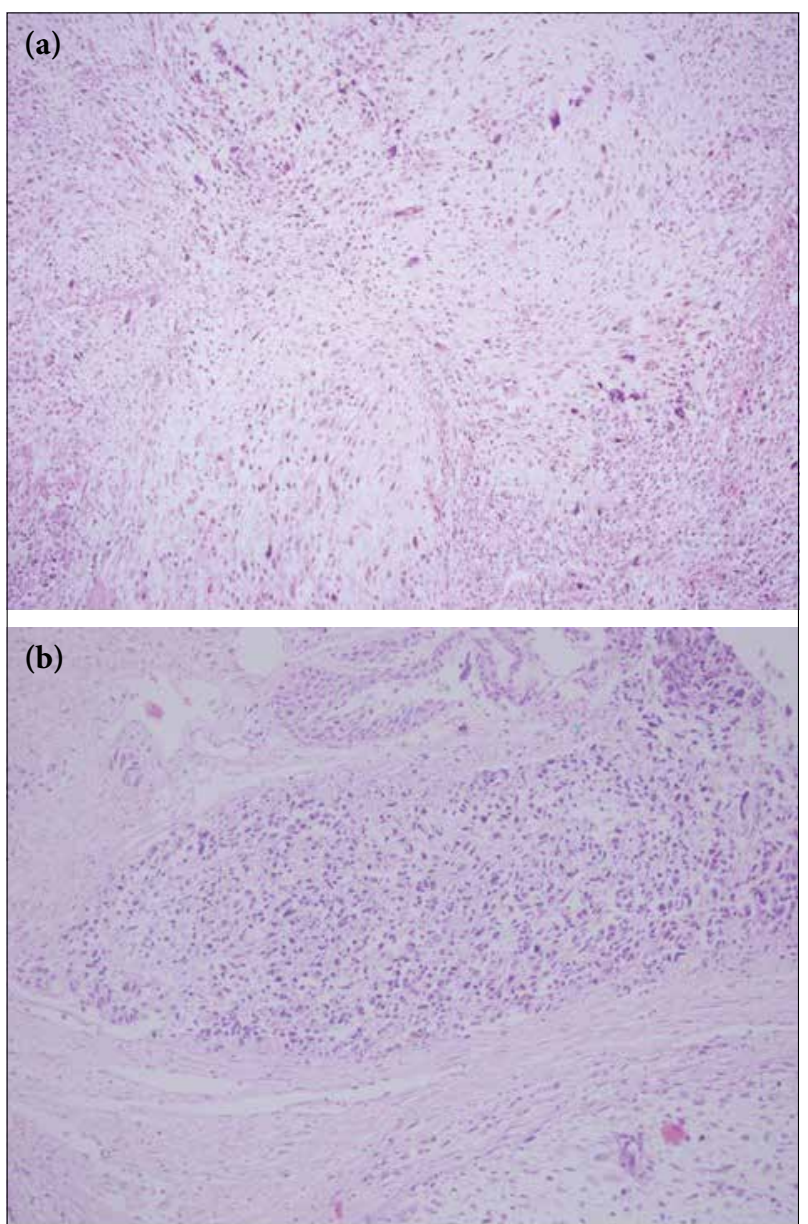

Fig. 1. (a) Atypical mesenchymal cells with rhabdomyoblastic differentiation. (b) Prostatic urethra and neoplasm infiltration.

\section{Case Report}

A 28-year-old male patient presented with the complaint of blood in urine. During rectal examination, external compression was detected in the ampulla; however, the mass in the lumen was not palpable. The pathology was not detected in the hematological and biochemical laboratory tests. On urinalysis, the total prostate-specific antigen (PSA) level of the patient was $1.25 \mathrm{ng} / \mathrm{mL}$ (range, 0-4 ng/mL ), and the other tumor markers were also normal. On abdominopelvic tomography, a $75 \times 41-\mathrm{mm}$ mass extending from the prostate to the left seminal vesicle was observed and another $94 \times 83-\mathrm{mm}$ mass was observed in the left pararectal area showing heterogeneous enhancement and pushing the sigmoid colon to the right lateral. No other metastasis was detected. A stenosis continuing along a $10-15 \mathrm{~cm}$ field was detected on rectosigmoidoscopy. A total prostatectomy and urinary diversion ileal loop surgery was performed. According
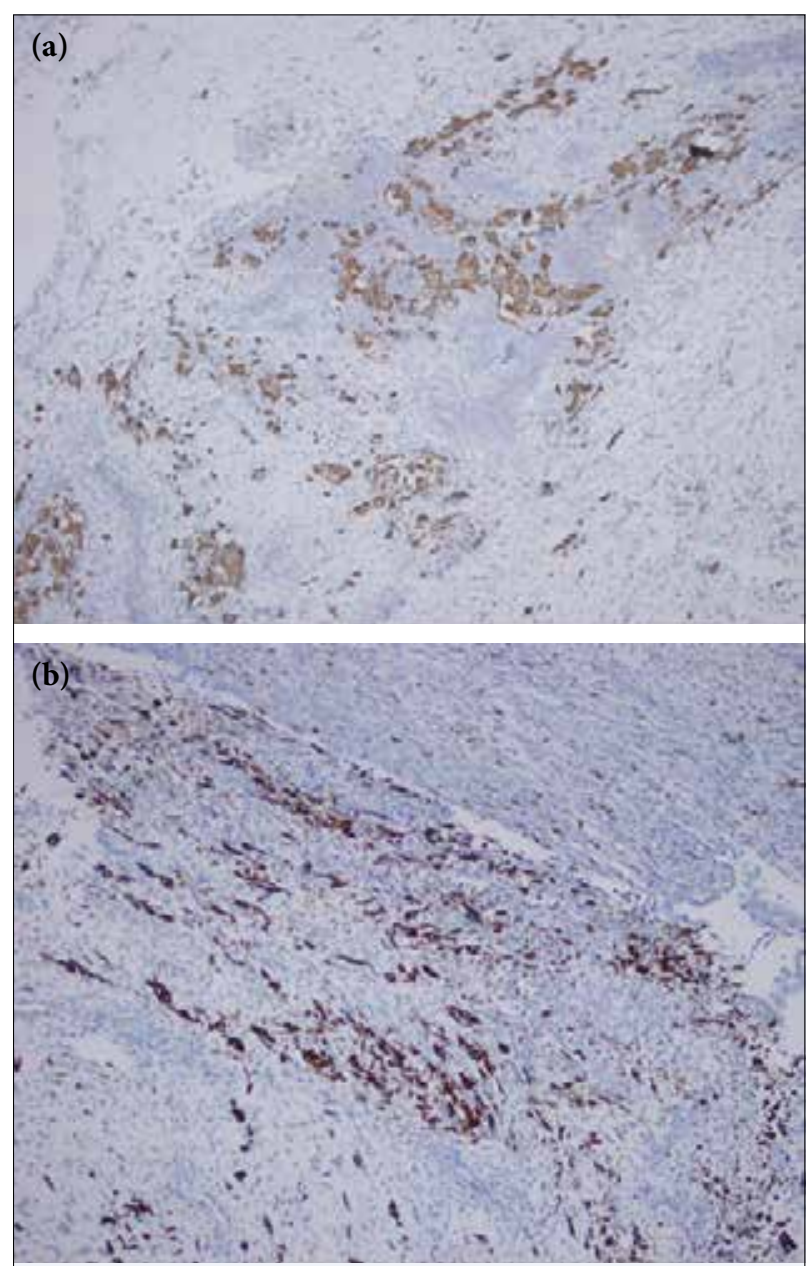

Fig. 2. (a) Muscle-specific actin expression of neoplastic cells. (b) Desmin expression of neoplastic cells.

to the pathological results, a high-grade malignant mesenchymal tumor showing rhabdomyoblastic differentiation, positive lymphovascular invasion, and positive left seminal vesicle invasion were obtained (Fig. 1a.b, 2a,b). Postoperative radiotherapy (46 Gy) followed by chemotherapy containing Adriamycin, ifosfamide, and mesna for 23 days was planned by the Oncology Council.

\section{Discussion}

Prostate develops from a diverticulum formed at the back wall of the primer urethra at the level where the Wolff channel opens into the cloaca and it consists of epithelial and stromal cells. The epithelial cells of the prostate consist of urothelial cells, secretory cells, basal cells, and neuroendocrine cells. [3,4] The secretory cells secrete PSA, prostatic acid phosphatase (PAP), acidic fluid, and other secretory products. The role of the basal cells is still not well understood, they do not color PSA or PAP, 
S-100 protein, and smooth muscle with actin. $[5,6]$ The neuroendocrine cells are the rarest observed cells within the prostatic epithelial cells, and even though their function is not known, they play the endocrine-paracrine regulatory role in the growth and development similar to the neuroendocrine cells in other organs. The stromal cells are striated and smooth muscle cells, which contain fibroblasts and neural and endothelial cells. [7]

The prostate tumors are classified according to WHO as follows: epithelial tumors, prostatic stromal tumors, hematolymphoid tumors, metastatic tumors, various tumors (such as cystadenoma, nephroblastoma, and germ cell tumors), and mesenchymal tumors (such as leiomyosarcoma, rhabdomyosarcoma, chondrosarcoma, angiosarcoma, malignant fibrous histiocytoma, malignant peripheral nerve sheath tumors, hemangioma, chondroma, leiomyoma, granular cell tumor, and solitary fibrous tumors).

More than $95 \%$ of the prostate cancers are formed by acinar adenocarcinomas showing differentiation in different degrees. In the diagnosis of the nonepithelial tumors of the prostate, it should be always considered whether the tumors are primary or secondary. In the prostate, the most common mesenchymal tumor is rhabdomyosarcoma during childhood, whereas in adults, it is leiomyosarcoma.

Malignant mesenchymal tumors were identified by Stout in 1948. [8] The prognosis of malignant mesenchymal tumors is controversial. Bradythe et al. reported in eight femoral and retroperitoneal cases that the survival rates for $2-3$ years were $75 \%$ and $37 \%$, respectively.[9] However, in a research conducted by Newman, Fletcher et al. on six cases, low-grade malignant behavior was identified in these tumors and the follow-up period of four of these cases was $<5$ years. [10] Adachia et al. determined that the presence of the rhabdomyosarcomas component and patients aged 40 years had bad prognosis and that sex, tumor area, tumor size or the MIB-1 classification index findings did not have an impact on the prognosis.[11] The presence of metastasis is reported to be a bad prognostic factor.

The soft tissue sarcomas of the prostate constitute $0.1 \%-0.2 \%$ of all primary prostate tumors. Prostate sarcomas, other than malignant mesenchymal tumor, reported in the literature are as follows: fibrosarcoma, malignant fibrous histiocytoma, angiosarcoma, and osteosarcoma. There are significant prognostic differences based on factors related to the tumors among the histological subtypes of the prostate sarcoma.

Unless focusing along on the adenocarcinoma, the serum PSA level in non-acinar originated tumors is not detected to be high. The imaging methods are not very helpful in differentiating the type of tumor and deter- mining whether it has malignant potential.

Since these cases are very rare, the treatment remains uncertain. Chemotherapy and radiotherapy is ineffective in some of the soft tissue sarcomas including malignant mesenchymal tumor. In recent studies, it was reported that the binary combinations of ifosfamide, cyclophosphamide, vincristine, doxorubicin, and dacarbazine doxorubicin increased the effectiveness of chemotherapy. There are studies indicating that in these cases, the multidisciplinary approach including surgery, radiotherapy, and chemotherapy might be useful.

Consequently, malignant mesenchymal tumors of the prostate are very rare. Although malignant mesenchymal tumors arising from primitive mesenchymal cells with totipotential differentiation capacity are considered to be high-degree malignant cancers, they are associated with the cellular elements, which are related to the malignant potential. These tumors may reach various sizes. Unlike epithelial tumors of the prostate, the serum PSA level remains normal in these cases. Biopsy with transrectal ultrasound is the main method for diagnosis. Since there are limited numbers of these cases in the literature, it is very difficult to correctly diagnose these cases because they require approaches different than those required for epithelial prostate cancer. In malignant mesenchymal tumors of the prostate, which require a multidisciplinary approach after radical prostatectomy, the implementation of a combination therapy including chemotherapy and radiotherapy is recommended.

\section{Disclosures Statement}

The authors declare no conflicts of interest.

Ethics Committee Approval: This study was conducted inaccordance with local ethical rules.

Peer-review: Externally peer-reviewed.

Conflict of Interest: None declared.

Authorship contributions: Concept - M. K; Design - E. K; Supervision - E. K; Materials - M. K; Data collection \&/ or processing - M. K; Analysis and/or interpretation - O. C. G; Literature search - A. Y; Writing - M. K; Critical review - S. M.

\section{References}

1. Paner GP, Aron M, Hansel DE, Amin MB. Non-epithelial neoplasms of the prostate. Histopathology 2012;60(1):166-86.

2. Ro JY, Amin MB, Kim KR, Ayala AG. Prostate and seminal vesicles. In: Fletcher CD, editor. Diagnostic Histopathology of Tumors, 3rd ed. Philadelphia: Churchill 
Livingstone Elsevier;2007. p. 798-800.

3. Epstein JI. The Prostate and Seminal Vesicles. In: Mills SE, editor. In: Diagnostic Surgical Pathology. 4th ed. New York: Raven Press 2004. p. 2083-132.

4. Signoretti S, Waltregny D, Dilks J, Isaac B, Lin D, Garraway $L$, et al. p63 is a prostate basal cell marker and is required for prostate development. Am J Pathol 2000;157(6):1769-75.

5. Young RH, Srigley JR, Amin MB, Ulbright TM, Cubilla AL. Tumors of the Prostate Gland, Seminal Vesicle, Male Urethra, and Penis. 3rd ed. Vol. 28. Washington, DC: Armed Forces Institute of Pathology;2000 p. 1-344.

6. Srigley JR, Dardick I, Hartwick RW, Klotz L. Basal epithelial cells of human prostate gland are not myoepithelial cells. A comparative immunohistochemical and ultrastructural study with the human salivary gland. Am J Pathol 1990;136(4):957-66.
7. Fraggetta F, Pepe P, Giunta ML, Aragona F. Primary high grade sarcoma of the specialised prostatic stroma: a case report with clinico-pathological considerations. Pathologica 2008;100(6):482-4.

8. Stout AP. Mesenchymoma, the Mixed Tumor of Mesenchymal Derivatives. Ann Surg 1948;127(2):278-90.

9. Brady MS, Perino G, Tallini G, Russo P, Woodruff JM. Malignant mesenchymoma. Cancer 1996;77(3):467-73.

10. Newman PL, Fletcher CD. Malignant mesenchymoma. Clinicopathologic analysis of a series with evidence of low-grade behaviour. Am J Surg Pathol 1991;15(7):60714.

11. Adachi T, Oda Y, Sakamoto A, Terashi T, Tamiya S, Hachitanda Y, et al. Prognostic factors in the socalled malignant mesenchymoma: a clinicopathological and immunohistochemical analysis. Oncol Rep 2003;10(4):803-11. 